\title{
Estimating the Semen Quality from Life Style Using Fuzzy Radial Basis Functions
}

\author{
Cemre Candemir
}

\begin{abstract}
Infertility is a major problem that directly affects many people worldwide. In recent years, fertility rates have decreased by up to $15 \%$ in young men. Changing living conditions, stress levels, environmental factors and nutritional habits play an important role in infertility. This paper suggests a novel method to estimate semen quality from lifestyle, environmental factors and daily habits using radial basis function neural networks. The accuracy of the suggested method was measured as $90 \%$. The results show that the proposed method can be applied as a practical, fast and cheap alternative method to laboratory tests.
\end{abstract}

Index Terms - Classification, fertility, fcm, fuzzy radial basis functions, neural networks.

\section{INTRODUCTION}

The American Society for Reproductive Medicine (ASRM) defines infertility as a disease of the reproductive system that disrupts the basic reproductive function of the human body [1]. According to the latest research data from the World Health Organization (WHO), which is carried out over a 20-year period (1990-2010), infertility affects approximately 48.5 million couples worldwide [2]. Although infertility is a critical component of the reproductive system both men and women, male infertility is given insufficient attention because maternal and child health is usually kept in the foreground [3]. However, being unable to have a child is a common problem for both sexes and infertility affects both men and women equally [4]. On the other hand, this problem may cause depression and anxiety as well as discrimination and exclusion by society [2]. In this respect, the underlying reasons for this problem need to be investigated correctly in term of both physical and environmental factors.

The fertility rate in men younger than 30 years has decreased worldwide by $15 \%$ [5]. In recent years, changing living conditions, intense work pressure and other environmental factors have a direct influence on male infertility as well as other factors, especially nutrition and daily habits. It is thought that factors such as increasing stress levels and nutritional disorders cause many physical and psychological problems as well as affecting fertility. In addition, besides physical and congenital anomalies, many other factors, such as using drugs, have a negative role in infertility. Pasqualotto et al. [6] listed these things as alcohol, cigarettes, narcotics (illicit drugs), anti-hypertensive drugs, calcium channel blockers, angiotensin converting enzyme

Manuscript received November 7, 2017; revised February 14, 2018.

C. Candemir is with the International Computer Institute at Ege University, 35100, Bornova, Izmir, Turkey (e-mail cemre.candemir@ege.edu.tr). inhibitors, psychotherapeutic agents, antipsychotics, antidepressants, chemotherapeutic agents, hormones, anabolic steroids and antibiotics. Other factors that play a role in infertility are related to occupational [7], environmental and/or lifestyle, where the possibility of testicular injury is high [8]. The risks related to the changing lifestyle over the last few years have arisen from mobile phones, electromagnetic radiation and high stress levels.

In this study, a method that uses a fuzzy radial basis functional neural network (FRBFNN) has been proposed to estimate semen quality. It is a fast, easy and scalable method that can be used to estimate the semen quality by profiling lifestyle and personal habits. Due to the fact that laboratory tests are generally expensive, the suggested method can be applied as a pre-test before laboratory testing. The paper is organized as follows: In section II, we summarize the related work. In section III, we give a background to the methods used for the proposed method. We describe the data set, details of the proposed method and the results in section four. In section five, the conclusions are given, which is followed by the reference list.

\section{RELATED WORK}

The basic function of an artificial neural network (ANN) is to mimic the human brain. An ANN is inspired by the human brain in the way it generalizes the events observed from examples, learning from the examples that it has never seen before, making decisions, classifying and optimizing. The abilities of an ANN are its main advantages when compared to traditional programming and statistical methods. ANNs are applicable in many areas as well as in the decision and classification problems found in medical and biomedical fields, such as the diagnosis of various diseases (including hypertension, cancer, rheumatic diseases, and vertigo), the analysis of medical images obtained by MRI and X-rays, and the prediction of a history of a disease [9]. Similarly, an ANN is the frequently used method in the diagnosis of prostate cancer [10]-[16], detecting changes in tumor and cell structures [17]-[22], tracking periodic differences in retinal images [23] and various urological dysfunctions [16], [24]-[28].

The earliest study on infertility using an ANN was performed with data obtained from hamsters [9]. In this study, the classification of the ANN was compared with linear discriminant function analysis (LDFA) and quadratic discriminant function analysis (QFDA). The study showed that an ANN is a powerful method for infertility analysis when compared to the statistical methods mentioned above. In another study that was performed using human data, an ANN and logistic regression were compared [29]. The results 
were parallel with the findings in [9].

When dealing with fertility data, several reasons can be given for the insufficiency of the statistical methods when compared to ANNs. The most important point is that semen analysis (SA), which is the main test of the male infertility potential, consists of several different parameters and the results of these tests are psychologically affected. The second major factor is that the data obtained from the patients are often segmented from multiple subjects and inconsistencies occasionally exist between these data [9]. In this respect, ANNs have a significant advantage over statistical methods because they are able to cluster large non-linear data sets flexibly and cost-effectively. Moreover, ANNs can make a predictive inference for clinical decisions with high accuracy and sensitivity. Another advantage is that they facilitate the dissemination of knowledge through rule inference [30].

With regard to this advantage of ANNs, Ma et al. [31] have shown that leptin can also be used diagnostically to predict male fertility with higher precision using neural networks.

Gil et al. [32] studied the effects of environmental factors and lifestyle on male fertility using artificial intelligence techniques and compared the decision tree, multi-layer perceptron (MLP) and support vector machine (SVM) methods with each other. It was observed that the MLP and SVM methods produce more accurate results than the decision tree method. It can be said that it is an effective alternative method in determining the factors that affect fertility.

\section{BACKGROUND}

\section{A. Fuzzy C-means algorithm}

In fuzzy clustering, unlike crisp clustering methods, an element may belong to more than one cluster with the same or different membership values at the same time, and the membership level can be adjusted precisely. In many cases, fuzzy clustering provides more accurate and natural clustering than classical clustering. Fuzzy C-means (FCM) is the most commonly used fuzzy clustering algorithm and aims to minimize the objective function defined in (1).

$$
J_{m}=\sum_{i=1}^{N} \sum_{j=1}^{c} u_{i j}^{m}|| x_{i}-c_{j} \|^{2} \quad, 1 \leq m<\propto
$$

where $X=\left[x_{i}\right]$ denotes the input vector, $U=\left[u_{i j}\right] \in$ $R^{c x n}\left(0 \leq u_{i j} \leq 1\right)$ denotes the membership degrees and $C=\left[c_{c}\right] \in R^{c x n}$ denotes the cluster centers.

\section{B. Radial basis function neural networks}

The radial basis function ( $\mathrm{RBF}$ ) was first used to solve a multivariable interpolation problem in [33] and initialized using ANNs in [34]. RBF neural networks (RBFNNs) are a special case of multi-layer perceptron and have two typical characteristics. The first one is, in contrast to the multi-layered perceptron, there is a single hidden layer and the other one is the use of radial basis functions as an activation function in the hidden layer. RBFNNs have many advantages according to the multi-layered neural network model because of their simple network architecture. The main advantage of RBFNNs is that they can be trained faster than other back-propagation algorithms.
As seen in Fig.1, the architecture of a RBFNN consists of three layers: The input, hidden and output layer. This simple structure can be configured to classify even high order non-linear patterns. This characteristic of the RBFNN is based on the Cover's theorem on the separability of the patterns [35]. According to Cover's theorem, the RBFNN solves the problem with linear models after linearize the complex structure of the input space, in other words, the high-order non-linear structures, by the non-linear transformations. The role of the radial basis function in this structure is to carry the input space to a high dimension space. The weight parameters correspond to the linear separation surface.

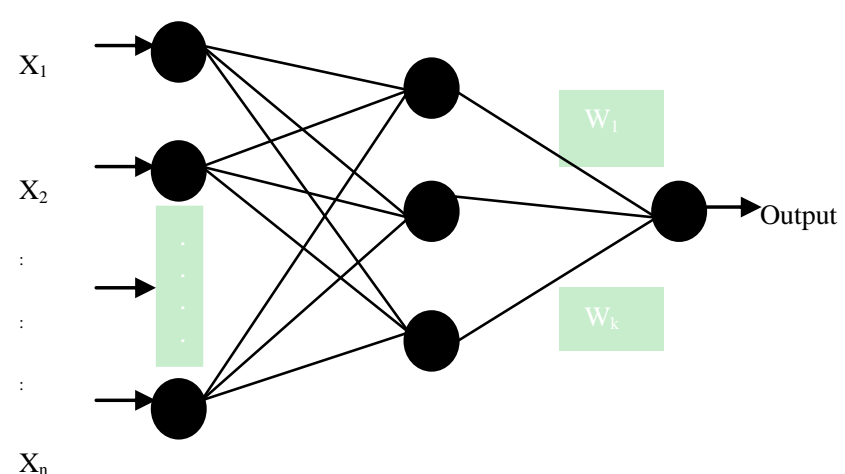

$$
\text { Input layer Hidden layer Output layer }
$$

In the input layej; ${ }^{1}$ wherhBEarthistecture the n-dimensional $\mathrm{X}$-vector, the input is transmitted to the hidden layer without preprocessing. In other words, all the weighted values between the input layer and the hidden layer are assumed to be " 1 ". Consequently, there is a significant reduction in the number of parameters to be changed during the learning process and the learning process is accelerated.

In hidden layer, each neuron is mathematically determined with a radial basis function. A radial function is a real-valued function that only depends on the distance to the origin or a center. Each function $\phi$ that provides $\phi(x)=\phi(|| x||)$ or $\phi(x, c)=\phi(|| x-c||)$ is a radial basis function. The generally known and used function is the Gaussian function that is defined as follows:

$$
\phi(x)=\phi\left(x-c_{i}\right)=\exp \left[-\frac{\left\|x-c_{i}\right\|^{2}}{2 \sigma_{i}^{2}}\right]
$$

Here, $x$ is the input vector and $c_{i}$ is the center vector. The output values of each neuron are calculated using (3), given below, and the response of the network is obtained by using these output values in the weighted sum in (4).

$$
\begin{aligned}
& \text { output }=\prod_{i=1}^{m} \psi\left(x_{i}, c_{i}, \sigma_{i}\right) \\
& y=\sum_{i=1}^{K} w_{i} o_{i}=\sum_{i=1}^{K}\left[w_{i} \prod_{j=1}^{m} \psi\left(x_{j}, c_{i j}, \sigma_{i j}\right)\right]
\end{aligned}
$$

Note that, the most important factor in the success of the $\mathrm{RBF}$ network is the selection of the appropriate radial-based function, which can be understood from (3). 


\section{Fuzzy radial basis function neural networks}

A fuzzy radial basis functions neural network (FRBFNN) is artificial neural network formed by bringing together RBF networks and the FCM algorithm [36]. By taking advantage of the fuzzification of the RBFNN, the model can handle different inputs (numerical or linguistic) and provide a smoother decision process if there are overlapping pattern classes in the output with membership values. The use of the FCM algorithm in the hidden layer for initializing the cluster centers allows some patterns belonging to one or more classes [36]. Thus, real-life situations are represented more accurately than in the classical crisp clustering methods, such as k-means. Cluster validity indexes are used to determine the optimal cluster numbers, i.e., the neuron numbers in the hidden layer.

Thw weights between the input and hidden layers are initialized by the cluster centers using FCM. In the known $\mathrm{RBF}$ architecture, even though the input vector does not match any pattern in the cluster center, the Gaussian distribution function is used, which allows the neuron to produce a non-zero response. The main goal in the FRBF network is to create a fuzzy partitioning that allows zero responses to be generated even if the input vector does not match any pattern in the hidden layer. This fuzzy partitioning is calculated using the membership function of the FCM. However, according to the structure of the function, the distance of a pattern to a cluster also depends on its distance to all the existing clusters. For this reason, it is necessary to change the known RBF architecture by adding another neuron $(\mathrm{H})$ to calculate the membership values locally using equation (5).

$$
h_{i}^{(j)}=\left(\frac{1}{d_{i j}}\right)^{2 / m-1}
$$

This auxiliary neuron $H$ is used to feed the total activation in the hidden layer to the output layer.

\section{PROPOSED METHOD}

\section{A. Data Set}

TABLE I: THE DESCRIPTION OF THE FEATURE VECTORS AND VALUES.

\begin{tabular}{|c|c|c|c|}
\hline \# & Variable Description & Values & Normalized \\
\hline 1 & $\begin{array}{l}\text { Season in which the } \\
\text { analysis was performed }\end{array}$ & $\begin{array}{l}\text { 1. Winter, 2. Spring, } \\
\text { 3. Summer, 4. Fall }\end{array}$ & $\begin{array}{l}(-1,-0.33 \\
0.33,1)\end{array}$ \\
\hline 2 & $\begin{array}{l}\text { Age at the time of } \\
\text { analysis }\end{array}$ & $18-36$ & $(0,1)$ \\
\hline 3 & $\begin{array}{l}\text { Childish diseases (ie, } \\
\text { chicken pox, measles, } \\
\text { mumps, polio) }\end{array}$ & 1. Yes, 2. No & $(0,1)$ \\
\hline 4 & $\begin{array}{lll}\begin{array}{l}\text { Accident } \\
\text { trauma }\end{array} & \text { or } & \text { serious } \\
\end{array}$ & 1. Yes, 2. No & $(0,1)$ \\
\hline 5 & Surgical intervention & 1. Yes, 2. No & $(0,1)$ \\
\hline 6 & $\begin{array}{l}\text { High fevers in the last } \\
\text { year }\end{array}$ & $\begin{array}{l}\text { 1. Less than three } \\
\text { months ago, } \\
\text { 2. More than three } \\
\text { months ago, } \\
\text { 3. No }\end{array}$ & $(-1,0,1)$ \\
\hline 7 & $\begin{array}{l}\text { Frequency of alcohol } \\
\text { consumption }\end{array}$ & $\begin{array}{l}\text { 1. Several times a } \\
\text { day, } \\
\text { 2. Every day, } \\
\text { 3. Several times a } \\
\text { week } \\
\text { 4. Once a week } \\
\text { 5. Hardly ever or } \\
\text { never }\end{array}$ & $\begin{array}{l}(0,0.2,0.4 \\
0.6,0.8,1)\end{array}$ \\
\hline
\end{tabular}

\begin{tabular}{|l|l|l|l|}
\hline$\#$ & \multicolumn{1}{|c|}{ Variable Description } & \multicolumn{1}{|c|}{ Values } & Normalized \\
\hline 8 & Smoking habit & $\begin{array}{l}\text { 1. Never } \\
\text { 2. Occasional } \\
\text { 3. Daily }\end{array}$ & $(-1,0,1)$ \\
\hline 9 & $\begin{array}{l}\text { Number of hours spent } \\
\text { sitting per day }\end{array}$ & $1-16$ & $(0,1)$ \\
\hline 10 & Output & $\begin{array}{l}\text { Diagnosis normal, } \\
\text { Altered }\end{array}$ & $\begin{array}{l}(\mathrm{N}) \\
(\mathrm{O})\end{array}$ \\
\hline
\end{tabular}

The "fertility" data was gathered using a questionnaire about daily habits with 100 healthy volunteers aged between 18 and 36 years old. The feature vectors in the data set were obtained by analyzing the questionnaire data using 9 variables according to the WHO 2010 criteria. A full description of the questionnaire and the other attributes can be found in [32]. The data set used in this study can also be reached from the Machine Learning Repository of California Irvine University (UCI) [37]. Table I shows the feature vectors of the full data set that will be used as the input vectors. The normalized values are written in the parenthesis.

\section{B. Methodology}

In this study, we present a novel method to estimate semen quality using an FRBF network. The whole algorithm can be viewed in Fig. 2.

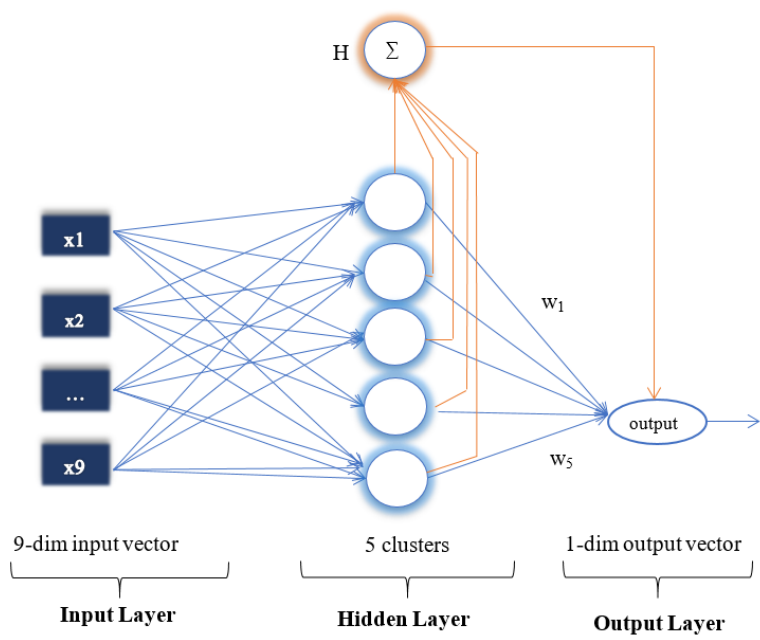

Fig. 2. The flowchart of the method.

The method consists of two main stages, a training stage and a testing stage. After selecting the parameters that train the FRBF network, the network will be tested with the selected parameters.

The process and training of the FRBF network consists of two phases. The first step is to find the center vectors (i.e., the number of hidden layer neurons) in the hidden layer and their propagation parameters. The second step is to determine the weights between the hidden layer and the output. In the proposed method, the weights were determined using the least mean squares method. Here, the aim is to adapt the weights and in this way, minimize the error function. In the first step, the center vectors can be selected manually, randomly or with the appropriate classification of the data set. The suitability of the classification is understood by the cluster validity index. There are various cluster validity index methods reported in the literature, such as partition coefficient, partition entropy, separation index, Fukuyama-Sugeno, Xie-Beni and partition coefficient and exponential separation (PCAES) [38]. In this study, the PCAES cluster validity index is used to determine the cluster 
number in the hidden layer and the optimal value was found to be 5 . The center vectors in the hidden layer are specified by the FCM algorithm. The optimal value of the fuzziness parameter $m$ was determined to be 2.0 in the range $[1.5,3.0]$ with increments of 0.1 .

\section{Performance and results}

In the proposed method, the classification using the FRBF network is performed in two stages, training and testing. Data from 100 people were randomly shuffled and the data set divided into two for these stages, 60 for the training stage and 40 for the testing stage. The number of hidden layer neurons was determined using the PCAES cluster validity index by running the FCM algorithm. The fuzziness number of the FCM was determined to be 2.0 in the range $[1.5,3.0]$ with increments of 0.1 . The proposed FRBF network architecture has [9xl] input vectors, 5 hidden layer neurons (center vectors) and a one-dimensional output vector that performs the binary classification. The overall architecture of the proposed FRBF method is given in Fig. 3.

The classification performance of the FRBF network in this architecture was measured to be $90 \%$. The optimal back-propagation training parameters are given in Table II.

\begin{tabular}{ll} 
TABLE II: THE BACK-PROPAGATION TRAINING PARAMETERS. \\
\hline \hline Parameters & Values \\
\hline Learning rate & 0.01 \\
Epochs & $50-100$ \\
Weight threshold & $1 \mathrm{E}-8$ \\
\hline \hline
\end{tabular}

It shows that the classification performance of the FRBF networks was very high according to the MLP, SVM and DT methods reported in the literature. The proposed method was compared with the other methods in Table III (PPV means the positive predicted values and NPV means the negative predicted values).

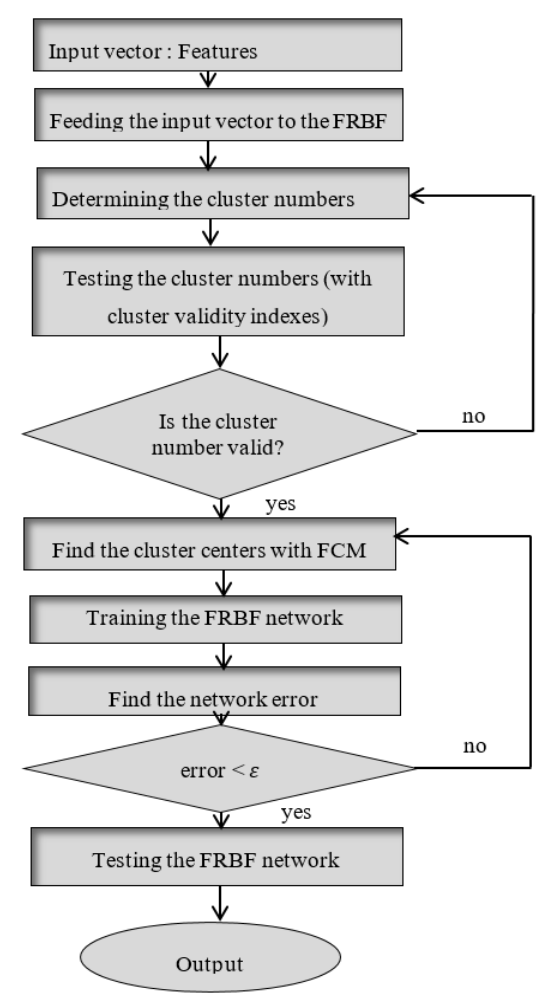

Fig. 3. The architecture of the proposed FRBF method.
TABLE III: THE PERFORMANCE OF THE PROPOSED METHOD.

\begin{tabular}{lllll}
\hline \hline & MLP & SVM & DT & FRBF \\
\hline Accuracy (\%) & 69.0 & 69.0 & 67.0 & $\mathbf{9 0 . 0}$ \\
Sensitivity (\%) & 72.8 & 73.9 & 71.7 & 92.1 \\
Specificity (\%) & 25.0 & 12.5 & 12.5 & 50.0 \\
PPV (\%) & 91.8 & 90.7 & 90.4 & 97.2 \\
NPV (\%) & 7.4 & 4 & 3.7 & 0.25 \\
\hline \hline
\end{tabular}

Due to its high accuracy over the other methods, FRBF can be used as an alternative pre-test before applying lab-tests to estimate semen quality.

\section{CONCLUSIONS}

In this study, we have proposed a novel method estimating semen quality from lifestyle, environmental factors and daily habits using radial basis function neural networks. The data was gathered using a questionnaire on the daily habits of 100 young and healthy volunteers. The feature vectors were determined by analyzing the questionnaire data with 9 variables according to the WHO 2010 criteria. This data set can also be reached from the Machine Learning Repository of California Irvine University (UCI). The proposed method consists of two stages including training and testing of the network. In the training step, the FCM algorithm was performed to determine the center vectors and a least mean square method was used to adjust the weights. The success of the method for estimating semen quality was determined to be $90 \%$. This result is more accurate when compared to the other methods used, which include MLP, SVM and DT. The results show that the proposed method can be applied as a practical, fast and cheap alternative method or pre-test before applying laboratory tests.

\section{ACKNOWLEDGMENTS}

The author would like to thank Prof. Dr. Aybars Uğur for his insightful comments on this research.

\section{REFERENCES}

[1] American Society for Reproductive Medicine | ASRM. [Online]. Available: http://www.asrm.org/detail.aspx?id=2322

[2] M. N. Mascarenhas, S. R. Flaxman, T. Boerma, S. Vanderpoel, and G A. Stevens, "National, regional, and global trends in infertility prevalence since 1990: A systematic analysis of 277 health surveys,' PLOS Med., vol. 9, no. 12, p. e1001356, Dec. 2012.

[3] W. Cui, "Mother or nothing: The agony of infertility," Bull. World Health Organ., vol. 88, no. 12, pp. 881-882, Dec. 2010.

[4] Infertility: Solutions for men and women. Cleveland Clinic. [Online]. Available:

https://my.clevelandclinic.org/health/transcripts/1362_infertility-solut ions-for-men-and-women

[5] J. A. Martin, B. Hamilton, P. D. Sutton, and S. J. Ventura, "Births: Preliminary data for 2006," Natl. Vital Stat. Rep., vol. 56, no. 7, 2007.

[6] F. F. Pasqualotto, A. M. Lucon, B. P. Sobreiro, E. B. Pasqualotto, and S Arap, "Effects of medical therapy, alcohol, smoking, and endocrine disruptors on male infertility," Rev. Hosp. Clínicas, vol. 59, no. 6, pp. 375-382, 2004.

[7] P. Claman, "Men at risk: Occupation and male infertility," Sex. Reprod. Menopause, vol. 2, no. 1, pp. 19-26, 2004.

[8] A. Agarwal, N. R. Desai, R. Ruffoli, and A. Carpi, "Lifestyle and testicular dysfunction: A brief update," Biomed. Pharmacother., vol. 62 , no. 8, pp. 550-553, Oct. 2008.

[9] D. J. Lamb and C. S. Niederberger, "Artificial intelligence in medicine and male infertility," World J. Urol., vol. 11, no. 2, pp. 129-136, May 1993.

[10] M. Gomari, P. Finne, T. Järvi, and U. H. Stenman, "Learning vector quantization, multilayer perceptron, neurofuzzy network and logistic 
regression in the diagnosis of prostate cancer," in Proc. 1998 Int. Conf. Parallel Distrib. Process. Tech. Appl., 1998, pp. 516-525.

[11] P. Finne et al., "Predicting the outcome of prostate biopsy in screen-positive men by a multilayer perceptron network," Urology, vol. 56, no. 3, pp. 418-422, Sep. 2000.

[12] T. Loch et al., "Artificial neural network analysis (ANNA) of prostatic transrectal ultrasound," The Prostate, vol. 39, no. 3, pp. 198-204, May 1999.

[13] A. L. Ronco and R. Fernandez, "Improving ultrasonographic diagnosis of prostate cancer with neural networks," Ultrasound Med. Biol., vol. 25, no. 5, pp. 729-733, Jun. 1999.

[14] T. Mattfeldt, H. Gottfried, and V. Schmidt, "Classification of spatial textures in benign and cancerous glandular tissues by stereology and stochastic geometry using artificial neural networks," vol. 198, no. 2 , pp. 143-158, Jan. 2000.

[15] R. J. Babaian et al., "Performance of a neural network in detecting prostate cancer in the prostate-specific antigen reflex range of 2.5 to 4.0 ng/mL," Urology, vol. 56, no. 6, pp. 1000-1006, Dec. 2000.

[16] D. Pantazopoulos, P. Karakitsos, A. Iokim-liossi, A. Pouliakis, E Botsoli-stergiou, and C. Dimopoulos, "Back propagation neural network in the discrimination of benign from malignant lower urinary tract lesions," J. Urol., vol. 159, no. 5, pp. 1619-1623, May 1998.

[17] A. Tewari and P. Narayan, "Novel staging tool for localized prostate cancer: a pilot study using genetic adaptive neural networks," J. Urol., vol. 160, no. 2, pp. 430-436, Aug. 1998.

[18] G. P. Murphy, P. b. Snow, J. Brandt, A. Elgamal, and M. K. Brawer, "Evaluation of prostate cancer patients receiving multiple staging tests, including ProstaScint ${ }^{\circledR}$ scintiscans," The Prostate, vol. 42, no. 2, pp 145-149, Feb. 2000

[19] J. T. Batuello et al., "Artificial neural network model for the assessment of lymph node spread in patients with clinically localized prostate cancer," Urology, vol. 57, no. 3, pp. 481-485, Mar. 2001.

[20] M. Han, P. B. Snow, J. M. Brandt, and A. W. Partin, "Evaluation of artificial neural networks for the prediction of pathologic stage in prostate carcinoma," Cancer, vol. 91, no. S8, pp. 1661-1666, Apr. 2001.

[21] A. Borque, G. Sanz, C. Allepuz, L. Plaza, P. Gil, and L. A. Rioja, "The use of neural networks and logistic regression analysis for predicting pathological stage in men undergoing radical prostatectomy: a population based study," J. Urol., vol. 166, no. 5, pp. 1672-1678, Nov. 2001.

[22] A. R. Zlotta, M. Remzi, P. B. Snow, C. C. Schulman, M. Marberger, and B. Djavan, "An artificial neural network for prostate cancer staging when serum prostate specific antigen is $10 \mathrm{ng} . / \mathrm{ml}$. or less," J. Urol., vol. 169, no. 5, pp. 1724-1728, May 2003

[23] C. Candemir, C. Çetinkaya, O. Kılınççeker, and M. Cinsdikici, "Vascular landmark classification in retinal images using fuzzy RBF," in Proc. 2013 21st Signal Processing and Communications Applications Conference (SIU), 2013, pp. 1-4.

[24] D. J. Bägli et al., "Artificial neural networks in pediatric urology: prediction of sonographic outcome following pyeloplasty," J. Urol. vol. 160, no. 3, Pt 2, pp. 980-983, discussion 994, Sep. 1998.

[25] G. S. Sonke, T. Heskes, A. L. Verbeek, J. J. de la Rosette, and L. A. Kiemeney, "Prediction of bladder outlet obstruction in men with lower urinary tract symptoms using artificial neural networks," J. Urol., vol. 163, no. 1, pp. 300-305, Jan. 2000.
[26] B. Kellner, C. G. Stief, H. Hinrichs, and C. Hartung, "Computerized classification of corpus cavernosum electromyogram signals by the use of discriminant analysis and artificial neural networks to support diagnosis of erectile dysfunction," Urol. Res., vol. 28, no. 1, pp. 6-13, Jan. 2000.

[27] D. Gil and M. Johnsson, "Using support vector machines in diagnoses of urological dysfunctions," Expert Syst. Appl., vol. 37, no. 6, pp. 4713-4718, Jun. 2010.

[28] D. Gil, M. Johnsson, J. M. Garcia Chamizo, A. S. Paya, and D. R. Fernandez, "Application of artificial neural networks in the diagnosis of urological dysfunctions," Expert Syst. Appl., vol. 36, no. 3, Part 2, pp. 5754-5760, Apr. 2009

[29] M. M. Samli and I. Dogan, "An artificial neural network for predicting the presence of spermatozoa in the testes of men with nonobstructive azoospermia," J. Urol., vol. 171, no. 6, Part 1, pp. 2354-2357, Jun. 2004.

[30] P. J. Lisboa and A. F. G. Taktak, "The use of artificial neural networks in decision support in cancer: A systematic review," Neural Netw., vol. 19, no. 4, pp. 408-415, May 2006.

[31] Y. Ma, B. Chen, H. Wang, K. Hu, and Y. Huang, "Prediction of sperm retrieval in men with non-obstructive azoospermia using artificia neural networks: leptin is a good assistant diagnostic marker," Hum. Reprod. Oxf. Engl., vol. 26, no. 2, pp. 294-298, Feb. 2011.

[32] D. Gil, J. L. Girela, J. De Juan, M. J. Gomez-Torres, and M. Johnsson, "Predicting seminal quality with artificial intelligence methods," Expert Syst. Appl., vol. 39, no. 16, pp. 12564-12573, Nov. 2012.

[33] M. J. D. Powell, "Radial basis function for multivariable interpolation a review," in Proc. IMA Conf. Algorithms Approx. Funct. Data, 1985.

[34] D. S. Broomhead and D. Lowe, "Multivariable Functional Interpolation and Adaptive Networks," Complex Syst., vol. 2, no. 3, pp. 321-355, 1988.

[35] S. Haykin, Neural Networks: A Comprehensive Foundation, 2nd ed. Upper Saddle River, NJ, USA: Prentice Hall PTR, 1998.

[36] S. Mitra and J. Basak, "FRBF: A Fuzzy Radial Basis Function Network," Neural Comput. Appl., vol. 10, no. 3, pp. 244-252, Dec. 2001.

[37] UCI Machine Learning Repository: Fertility Data Set. [Online] Available: http://archive.ics.uci.edu/ml/datasets/Fertility\#Bardan R

[38] K.-L. Wu and M.-S. Yang, "A cluster validity index for fuzzy clustering," Pattern Recognit. Lett., vol. 26, no. 9, pp. 1275-1291, Jul. 2005 .

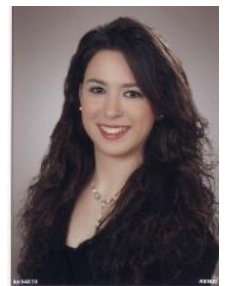

Cemre Candemir is a $\mathrm{Ph} . \mathrm{D}$. candidate and research assistant at the International Computer Institute in Ege University, Turkey. She received her M.Sc. degree in Information Technologies from the same Institute in 2012 and B.Sc. degree in Mathematics from Ege University in 2009. Her research interests concentrate on medical imaging, artificial neural networks and fmri studies. 